Palavras chave: Motosserra

Corte florestal Estudo de tempos

Histórico: Recebido 20/09/20 I I Aceito 29/04/2014

Keywords: Chainsaw Forest harvest Time study

Correspondência: elton@ufrb.edu.br

$\mathrm{DOI}$
Elton da Silva Leite', Haroldo Carlos Fernandes², Ilvânio Luis Guedes³ , Edvaldes José do Amaral $^{3}$

\section{ANÁLISE TÉCNICA E DE CUSTOS DO CORTE FLORESTAL SEMIMECANIZADO EM POVOAMENTOS DE EUCALIPTO EM DIFERENTES ESPAÇAMENTOS}

RESUMO: Objetivou-se, com este trabalho, avaliar, tecnicamente, os custos do corte florestal semimecanizado, em plantios de eucalipto de primeira rotação, em declividade média de $26,22^{\circ}(58,2 \%)$, nos espaçamentos $3 \times 2,5 \mathrm{~m}, 3 \times 3,33 \mathrm{~m} \mathrm{e} 3 \times 4 \mathrm{~m}$, com volumes de $0,326,0,36 \mathrm{I}$ e $0,370 \mathrm{~m}^{3}$ por árvore, no sistema I + I (um operador de motosserra e um ajudante). Os resultados mostraram que as atividades que consomem mais tempo no corte e processamento com a motosserra Stihl MS360 são: deslocamento, traçamento e $1^{\circ}, 2^{\circ}$ e $3^{\circ}$ corte. No espaçamento $3 \times 2,5 \mathrm{~m}$, a produtividade média foi de $4,69 \mathrm{~m}^{3} \cdot \mathrm{h}^{-1}$, a um custo de produção de $5,16 \mathrm{R} \$ \cdot \mathrm{m}^{-3}$. Para o espaçamento $3 \times 3,33 \mathrm{~m}$, foi de $5,19 \mathrm{~m}^{3} \cdot \mathrm{h}^{-1}$ e 4,66 R\$. $\mathrm{m}^{-3}$ e para o espaçamento $3 \times 4 \mathrm{~m}$, foi de $5,73 \mathrm{~m}^{3} \cdot \mathrm{h}^{-1}$ e $4,22 \mathrm{R} \$ \cdot \mathrm{m}^{-3}$. O item de maior influência nos custos operacionais foi a mão de obra, com $75 \%$ dos custos totais. O espaçamento maior resultou em maior produtividade e menores custos de produção.

\section{TECHNICAL AND ECONOMIC ANALYSIS OF SEMIMECHANIZED HARVEST OF EUCALYPTUS IN DIFFERENT SPACING}

ABSTRACT: The objective of this study was to evaluate technically semi-mechanized forest cutting costs in eucalypt plantations in first rotation, average slope of $26.22^{\circ}$ (58.2\%), spacing $3 \times 2.5 \mathrm{~m}, 3 \times 3.33 \mathrm{~m}$ and $3 \times 4 \mathrm{~m}$, with volumes of $0,326,0,36 \mathrm{I}$ and $0,370 \mathrm{~m}^{3}$ per tree, in the I $+\mathrm{I}$ system (one chainsaw operator and a helper). The results showed that the activities that consume more time in cutting and processing with the chainsaw Stihl MS360 are: displacement, bucking and Ist, 2nd and 3rd cut. Spaced at $3 \times 2.5 \mathrm{~m}$, average productivity of was $4.69 \mathrm{~m}^{3} \cdot \mathrm{h}^{-1}$ at a production cost of $5.16 \mathrm{R} \$ \cdot \mathrm{m}^{-3}$. In the $3 \times 3.33 \mathrm{~m}$ spacing average productivity was $5.19 \mathrm{~m}^{3} \cdot \mathrm{h}^{-1}$ and $4.66 \mathrm{R} \$ \cdot \mathrm{m}^{-3}$ and in the $3 \times 4 \mathrm{~m}$ spacing was $5.73 \mathrm{~m}^{3} \cdot \mathrm{h}^{-1}$ and $4.22 \mathrm{R} \$ \cdot \mathrm{m}^{-3}$. The item of greatest influence on operating costs was labor, with $75 \%$ of total costs. The wider spacing resulted in higher productivity and lower production costs.

' Universidade Federal do Recôncavo da Bahia - Cruz das Almas, Bahia, Brasil

2 Universidade Federal de Viçosa - Viçosa, Minas Gerais, Brasil

${ }^{3}$ CENIBRA - Belo Oriente, Minas Gerais, Brasil 


\section{INTRODUÇÃO}

Embora as máquinas florestais sejam produtivas, estão sujeitas a fatores ambientais que podem restringir seu uso, como: densidade do talhão, topografia, tipo de solo, volume por árvore e distância de transporte (MACHADO et al., 2008). A motosserra caracteriza-se por operar em diversas condições do ambiente e, muitas vezes, de difícil acesso, com baixo custo de operação, podendo realizar o corte e o processamento das árvores em qualquer tipo de terreno. Permite, ainda, que se atinja produtividade individual relativamente elevada, com baixo investimento inicial (SANT'ANNA; MALINOSKI, 2002).

O corte florestal com motosserra é efetuado, em sua grande maioria, com motosserras de diversas marcas, modelos e tamanhos, muitas vezes sem presença de todos os dispositivos necessários à segurança do operador (SANT'ANNA, 2008).

A produtividade da motosserra é fortemente influenciada, principalmente, pelo espaçamento de plantio e declividade do terreno. A quantidade de árvores por área e sua distribuição podem modificar as condições ambientais dos povoamentos florestais e a partição dos fatores de produção, afetando a produtividade, as características da madeira e os custos de produção (PASSOS et al., 2006).

A escolha do espaçamento tem como objetivo inicial proporcionar, para cada árvore, espaço suficiente para se obter o crescimento máximo, com melhor qualidade e menor custo nas operações (SILVA et al., 20I0).

Objetivou-se, com este trabalho, avaliar tecnicamente e os custos do corte florestal semimecanizado, em plantios de eucalipto nos espaçamentos $3 \times 2,5 \mathrm{~m}, 3 \times 3,33 \mathrm{~m}$ e $3 \times 4 \mathrm{~m}$.

\section{MATERIAL E MÉTODOS}

\section{Caracterização da área de estudo}

A área de estudo era composta por povoamentos híbridos clonais de Eucalyptus urophila $x$ Eucalyptus grandis, primeira rotação, com sete anos de idade, pertencente à Celulose Nipo Brasileira S.A. CENIBRA, localizada na região do Rio Doce, no município de Belo Oriente - MG.

A coleta de dados foi de 02 a 29 de junho de 2009, no turno de operação, de $8 \mathrm{~h}$ às $16 \mathrm{~h}$, em solo classificado de Cambissolo Latossólico e clima temperado chuvoso-mesotérmico, com precipitação média anual de $1.163 \mathrm{~mm}$, temperatura média anual de $25,2^{\circ} \mathrm{C}$, média das temperaturas máximas de $31,5^{\circ} \mathrm{C}$, médias das temperaturas mínimas de $19,1^{\circ} \mathrm{C}$ e umidade relativa média de $65,2 \%$.

A declividade média local foi de $26,22^{\circ}$ $(58,2 \%)$, com máxima de $35,1^{\circ}(78,0 \%)$, mínima de $20,4^{\circ}(45,3 \%)$ e coeficiente de variação $17,86 \%$, determinado com o auxílio de um inclinômetro digital Haglôf Sweden Profissional.

As unidades amostrais foram inventariadas individualmente e foram obtidas as médias das árvores (árv) por área e volumes $\left(\mathrm{m}^{3}\right)$, por hectare (ha) e por árvore (árv), como se evidencia na Tabela I.

TABELA I Características dendrométricas do povoamento de eucalipto.

TABLE I Dendrometric characteristics of the eucalyptus stand.

\begin{tabular}{ccccc}
\hline \multirow{2}{*}{ Espaçamento Variáveis } \\
\cline { 2 - 5 } & árv·ua $^{-1}$ & árv·ha & $\mathrm{m}^{3} \cdot \mathrm{ha}^{-1}$ & $\mathrm{~m}^{3} \cdot$ árv $^{-1}$ \\
\hline $3 \times 2,5 \mathrm{~m}$ & 118,333 & 1315,106 & 428,800 & 0,326 \\
$3 \times 3,33 \mathrm{~m}$ & $86,111 \mathrm{1}$ & 963,993 & 347,930 & 0,361 \\
$3 \times 4 \mathrm{~m}$ & 79,636 & 887,112 & 328,470 & 0,370 \\
\hline
\end{tabular}

árv = árvores; ua $=$ unidade amostral; ha $=$ hectare; $\mathrm{m}^{3}=$ metro cúbico.

\section{Descrição e operação da motosserra}

Utilizou-se motosserra da marca STIHL, modelo MS360, com motor dois tempos, potência de $3,5 \mathrm{~kW}$ (5,3 hp) e 6I,5 cilindradas, peso de 5,7 kg e sabre com comprimento de 13 polegadas $(0,325 \mathrm{~m})$.

O corte das unidades experimentais foi iniciado pela parte mais baixa do terreno, em uma faixa de corte de quatro linhas. Inicialmente, realizava-se $\circ$ corte e processamento das árvores da $\mathrm{I}^{\mathrm{a}}$ e $4^{\mathrm{a}}$ linhas. Posteriormente, realizou-se a mesma sequência de operações para a $2^{\mathrm{a}}$ e $3^{\mathrm{a}}$ linhas.

No decorrer da operação, foi utilizada uma equipe de trabalho representada pelo sistema I + I (um operador de motosserra e um ajudante), com traçamento de 2,60 m. Empregou-se sempre a mesma equipe de trabalho, sendo que, todos eram treinados na função e possuíam experiência de mais de dois anos no sistema adotado.

\section{Análise de tempos e movimentos}

Para tabular o ciclo operacional, realizouse $\circ$ estudo de tempos e movimentos, pela divisão das atividades executadas durante as operações. As atividades foram definidas com observações visuais, e a cada I5 segundos eram descritas no formulário. 
Os elementos parciais que compuseram as atividades operacionais foram divididos em duas etapas: o corte e o processamento, como descritos na Tabela 2. Foram registradas todas as ocorrências da jornada, tais como: horas disponíveis, horas trabalhadas, consumo de combustível, tempo parado, etc. Foram incluídos, ainda, detalhes do local de trabalho; inclinação da unidade experimental, condições climáticas, condições operacionais, etc.

TABELA 2 Tempos técnicos referentes às operações de corte e processamento no sistema semimecanizado.

TABLE 2 Technical times for the operations of cutting and processing in the semimechanized system.

\section{Corte}

Deslocar com máquina: tempo em que o operador transitou de uma árvore a outra pela parcela.

Limpar pé da árvore: período em que o operador limpa a base da árvore para realizar o corte.

Posicionar para corte: período em que o operador planejou a derrubada da árvore e posicionou-se para efetuar os cortes de abate da árvore.

$1^{\circ}, 2^{\circ}$ e $3^{\circ}$ cortes: uso da motosserra para realização dos cortes para abate das árvores.

Empurrar/tombar árvore: tempo em que o operador/ ajudante empurra a árvore para 0 tombamento, direcionando-se a sua queda.

Sair para área de segurança: deslocamento do operador/ ajudante juntamente com a máquina para local seguro.

Pausa técnica: tempo gasto com ajuste de corrente, comunicação via rádio, etc.

Pausa pessoal: período referente às necessidades fisiológicas do operador/ajudante.

Outros: tempo para tombar árvores entrelaçadas de espera para melhorias climáticas (vento) para efetuar a derrubada.

Processamento

Deslocar com a máquina: período de deslocamento entre os pontos de seccionamento das árvores.

Posicionar: tempo de posicionamento do operador para efetuar traçamento.

Traçar: acionamento do componente cortante da motosserra até o seccionamento do tronco.

Desgalhar: tempo em que o operador retirou os galhos remanescentes da desgalha com a machadinha.

Medir torete: medição dos toretes ao longo da árvore, com o auxilio de uma vara (tipo "de pesca") de 2,60 m de comprimento.

Pausa técnica: tempo gasto com ajuste de corrente, comunicação via rádio, etc.

Pausa pessoal: período referente às necessidades fisiológicas do operador/ajudante.

Outro: período de retirada de toretes para desobstruir pontos de traçamento.

\section{Produtividade}

A estimativa da produtividade do equipamento é obtida, por meio do volume de madeira da unidade experimental e as, horas efetivamente trabalhadas (SILVA et al., 20I0). Segundo os autores, a eficiência operacional é a porcentagem do tempo efetivamente trabalhado.

\section{Análise de custo}

O custo operacional total é obtido pela soma dos custos fixos e variáveis da máquina analisada.

\section{Custos fixos (CF)}

Os custos fixos correspondem aos fatores físicos de produção, e são determinados pela somatória dos custos de depreciação, juros, seguros e impostos, abrigo e taxas de administração.

\section{a) Depreciação (Dp)}

Depreciação é o custo decorrente do desgaste ou obsolescência natural da máquina, ao longo do tempo, no processo produtivo. $O$ custo da depreciação foi determinado pelo método linear, expresso pela equação I (AMERICAN SOCIETY OF AGRICULTURAL ENGINEERS - ASAE, 200I), sendo $\mathrm{Va}=$ valor de aquisição ( $R \$ 1.632,00) ; \mathrm{Vr}=$ valor residual $(\mathrm{R} \$ 195,84$, $12 \%$ de $\mathrm{Va}$ ); $\mathrm{N}=$ vida útil estimada (2,53 anos); e he = horas efetivas de uso anual ( $1.770,83 \mathrm{~h})$.

$\mathrm{Dp}=\left(\frac{\mathrm{Va}-\mathrm{Vr}}{\mathrm{N} \cdot \mathrm{he}}\right)$

\section{b) Juros, seguros e impostos (JSI)}

Os juros, seguros e impostos (JSI) foram calculados sobre o valor da motosserra, aplicados ao investimento médio anual (IMA) (CANTO, 2009). Utilizou-se uma taxa de juros de $12 \%$ a.a. Os custos de JSI estão representado pela equação 2 , sendo IMA (equação 3 ) = investimento médio anual $(R \$) ; i=$ taxa de juros, mais seguros, anuais simples (I2\%); e N = vida útil estimada (anos).

$$
\begin{aligned}
& J S I=\frac{I M A \cdot i}{h e} \\
& I M A=\frac{(V a-V r) \cdot(N+I)}{2 \cdot N}+V r
\end{aligned}
$$

\section{c) Abrigo (CA)}

O valor do abrigo é calculado e pago em função do local de armazenamento requerido, apresentado na 
equação 4. Considerou-se um fator de ajuste (FA) de I,5\%.

$C A=\frac{(V a \cdot F A)}{h e}$

\section{d) Taxas de administração (T)}

É calculada em função dos encargos administrativos. Foi considerado um percentual do valor da máquina, de acordo com o preconizado por ASAE (200I). Dessa forma, o fator de ajuste (FA) foi de $10 \%$ $(0, I)$, tal qual equação 5 .

$\mathrm{T}=\frac{\mathrm{Va} \cdot \mathrm{FA}}{\mathrm{he}}$

\section{Custos variáveis (CV)}

Os custos variáveis correspondem a todos os fatores variáveis de produção. Esses custos variam em função dos custos de combustíveis, lubrificantes de corrente, manutenção, reparos, transporte de pessoal e maquinário.

\section{a) Custo de combustível (CC)}

Refere-se ao custo de consumo de combustível (mistura de gasolina e óleo dois tempos) as máquinas, expresso pela equação 6 (CANTO, 2009; SILVA, 2008), sendo $\mathrm{Pu}=$ preço do litro de combustível (gasolina + óleo dois tempos) (4,13 R\$. $\left.\mathrm{L}^{-1}\right)$; e $\mathrm{c}=$ consumo de combustível por hora efetiva $\left(0,76 \mathrm{~L} \cdot \mathrm{h}^{-1}\right)$.

$\mathrm{CC}=\mathrm{Pu} \cdot \mathrm{C}$

\section{b) Custo de lubrificantes de corrente (Clc)}

Esse custo é referente ao consumo de óleo lubrificante de corrente do sistema de corte da motosserra, e foi calculado pela equação 7 (SILVA, 2008) com base no percentual de combustível gasto pela máquina, sendo $\mathrm{Clc}$ $=$ custo de lubrificantes de corrente $\left(\mathrm{R} \$ \cdot \mathrm{h}^{-1}\right) ; \mathrm{PI}=$ preço por litro do lubrificante $\left(2,48 \mathrm{R} \$ \cdot \mathrm{L}^{-1}\right)$; e $\mathrm{cl}=$ consumo de lubrificantes por hora efetiva $\left(0,27 \mathrm{~L} \cdot \mathrm{h}^{-1}\right)$.

$\mathrm{Clc}=\mathrm{Pl} \cdot \mathrm{cl}$

\section{c) Custo de reparos e manutenção (CRM)}

Corresponde aos custos destinados aos reparos e manutenção da máquina durante sua vida útil, conforme expresso pela equação 8 .

$\mathrm{CRM}=\frac{\mathrm{Va}}{\mathrm{he}}$

\section{d) Custo de mão de obra (CMO)}

Refere-se ao custo total dos operadores, como o salário mensal, $13^{\circ}$ salário, férias, benefícios, encargos sociais e seguros, tal qual demonstrado pela equação 9 , sendo $\mathrm{CMO}=$ custo de mão de obra $\left(\mathrm{R} \$ \cdot \mathrm{h}^{-1}\right)$; Soa $=$ operador de motosserra e ajudante (R\$I.400,00); s = encargos sociais $(0,77) ; M=$ meses no ano ( 12 messes). Para efeito de cálculo, adotou-se o fator de encargos sociais de $77 \%$ sobre o salário.

$\mathrm{CMO}=\frac{\text { Soa } \cdot M \cdot(I+s)}{\text { he }}$

\section{e) Custo de transporte de pessoal e maquinário (CTPM)}

Refere-se ao custo do deslocamento de pessoal até o local das operações. Esse custo foi obtido a partir dos dados da empresa, em valores mensais, pela quantidade de horas trabalhadas (MOREIRA, 2000).

\section{Custo de produção}

O custo de produção da máquina, expresso em $\mathrm{R} \$ \cdot \mathrm{m}^{-3}$, foi estimado pela relação entre custos operacionais $\left(R \$ \cdot h^{-1}\right)$ e a produtividade $\left(\mathrm{m}^{3} \cdot \mathrm{h}^{-1}\right)$. Utilizouse a taxa de câmbio, Dólar (R\$2,208), cotados em 02 de outubro de 2013.

\section{Delineamento estatístico}

Utilizou-se o Delineamento Inteiramente Casualizado (DIC), composto por sete repetições e três tratamentos (espaçamentos $3 \times 2,5 \mathrm{~m}, 3 \times 3,33 \mathrm{~m}$ e $3 \times$ $4 \mathrm{~m})$. Os sistemas foram submetidos à significância de $5 \%$ de probabilidade. Para a análise de contraste entre os tratamentos foi aplicado o teste de Tukey, ao nível de $5 \%$ de probabilidade.

\section{RESULTADOS E DISCUSSÃO}

\section{Estudo de tempos e movimentos}

O tempo médio para corte e processamento com a motosserra nos espaçamentos $3 \times 2,5 \mathrm{~m}$ e $3 \times$ $3,33 \mathrm{~m}$ foram 25 e $11 \%$, respectivamente, maiores que no espaçamento $3 \times 4 \mathrm{~m}$. No espaçamento $3 \times 4$ $\mathrm{m}$ gastou-se $62 \mathrm{l} \mathrm{s} \cdot \mathrm{m}^{-3}$ de madeira cortada e processada. Já, os espaçamentos $3 \times 2,5$ m e $3 \times 3,33$ consumiram, respectivamente, 792 e $699 \mathrm{~s} \cdot \mathrm{m}^{-3}$. Na Tabela 3, estão apresentados os percentuais das atividade de corte e processamento para os espaçamentos. 
TABELA 3 Percentual das atividades de corte e processamento com a motosserra em função dos espaçamentos.

TABLE 3 Percentage of cutting and processing activities with the chainsaw as a function of spacing.

\begin{tabular}{ccc}
\hline Espaçamento & Corte (\%) & Processamento (\%) \\
\hline $3 \times 2,5 \mathrm{~m}$ & 41,08 & 58,92 \\
$3 \times 3,33 \mathrm{~m}$ & 40,92 & 59,08 \\
$3 \times 4 \mathrm{~m}$ & 41,33 & 58,67 \\
\hline
\end{tabular}

Em trabalho conduzido por Mousavi (2009), avaliando o corte com motosserra Stihl em uma floresta nativa de diversos volumes por árvore, DAP e comprimento de toretes no norte do Iran, concluíram que a etapa de corte consumiu maior tempo (58\%), valores diferentes dos encontrados neste trabalho. Esse fato pode ser explicado em virtude da densidade da madeira e dos maiores diâmetros das árvores, esses eventos aumentam o tempo de corte.
Na Figura I, são apresentadas as atividades operacionais da etapa de corte e, na Figura 2, as atividades operacionais de processamento com motosserra. Desloca-se com a máquina e $1^{\circ}, 2^{\circ}$ e $3^{\circ}$ cortes foram atividades que consumiram maior tempo, gastando 22 e $40 \%$ da etapa de corte. Já, na etapa de processamento, as atividades mais expressivas foram deslocar com a máquina e traçar, consumindo 28 e $42 \%$ do tempo.

\section{Produtividade}

$\mathrm{Na}$ Tabela 4, estão apresentados os dados de produtividade em relação ao espaçamento. Os dados atenderam aos requisitos: normalidade e homogeneidade das variâncias. Os resultados do teste Tukey apresentaram diferenças entre os espaçamentos, ao nível de $5 \%$ de probabilidade. Observa-se que a produtividade média

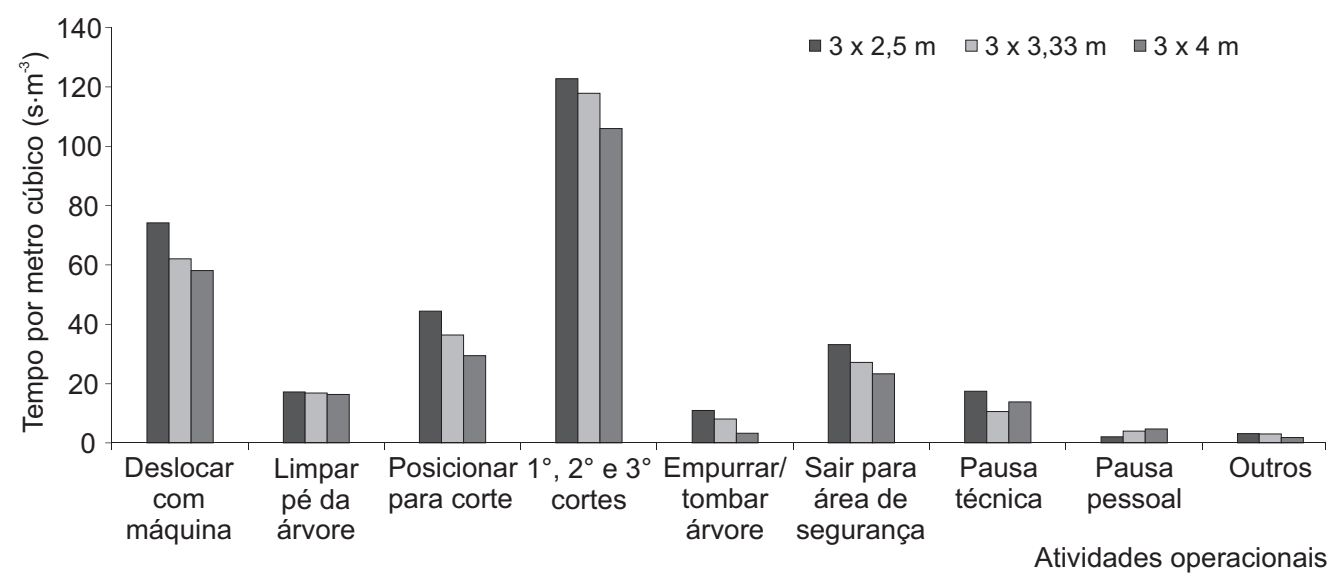

FIGURA I Atividades operacionais do corte no sistema semimecanizado.

FIGURE I Operational activities of the cutting in the semimechanized system.

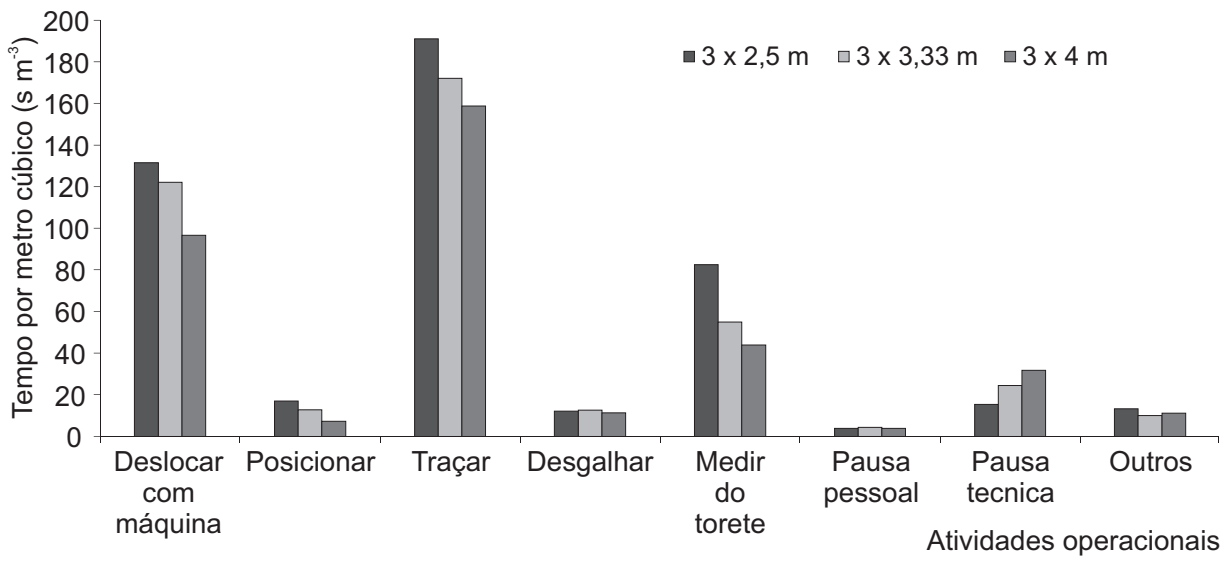

FIGURA 2 Atividades operacionais do processamento no sistema semimecanizado.

FIGURE 2 Operational activities of the processing in the semimechanized system. 
da equipe tende a ser maior quando se aumenta o espaçamento, pois há correlação positiva com o volume por árvore, maiores espaçamentos tendem a apresentar maior volume por árvore. Em média, o espaçamento $3 \times 4 \mathrm{~m}$ foi superior $10 \%$ e $22 \%$, respectivamente, em produtividade, que nos espaçamentos $3 \times 2,5 \mathrm{~m}$ e $3 \times 3,33 \mathrm{~m}$.

TABELA 4 Análise estatística da produtividade no sistema semimecanizado em relação aos espaçamentos de plantios.

TABLE 4 Statistical analysis of the productivity in the semimechanized system in relation to planting spacing.

\begin{tabular}{|c|c|c|c|c|c|}
\hline \multicolumn{6}{|c|}{ Anova (teste F) } \\
\hline FV & GL & SQ & QM & Fc & $p$ \\
\hline Espaçamentos & 2 & 3,780 & 1,890 & $15,038 *$ & 0,00014 \\
\hline Resíduo & 18 & 2,262 & 0,126 & & \\
\hline Total & 20 & 6,041 & & & \\
\hline \multicolumn{6}{|c|}{ Teste de média (Tukey 5\%) } \\
\hline \multirow{2}{*}{ Espaçamentos } & \multicolumn{5}{|c|}{ Produtividade média } \\
\hline & & $\cdot h^{-1}$ & $\mathrm{~m}^{3} \cdot \mathrm{dia}^{-1}$ & $\mathrm{Di}$ & ferenças \\
\hline $3 \times 2,5 \mathrm{~m}$ & & 69 & 34,14 & & $\mathrm{a}$ \\
\hline $3 \times 3,33 m$ & & 19 & 37,56 & & $b$ \\
\hline $3 \times 4 \mathrm{~m}$ & & 73 & 40,73 & & c \\
\hline
\end{tabular}

Os valores de produtividade encontrados neste trabalho são maiores que os encontrados por Santos et al. (2000). Esse fato pode ser explicado pela modernização e diferenças entre as motosserras, equipe de trabalho e, ou, pelo maior volume das árvores deste estudo.

\section{Análise de custo}

O custo operacional do sistema semimecanizado foi de $24,20 \mathrm{R} \$ \cdot \mathrm{h}^{-1}$, para todos os espaçamentos. Os custos fixos foram de $0,99 \mathrm{R} \$ \cdot \mathrm{h}^{-1}$ e os custos variáveis foram de 23,22 R\$· $h^{-1}$. Na Figura 3, são apresentados os valores correspondentes aos itens que compõem o custo operacional, com taxa real de juros de $12 \%$ aa. e eficiência operacional de $90,2 \%$. Os custos com mão de obra foram os mais onerosos no valor final com $75 \%$, valores similares aos resultados encontrados por Andreon (20II).

\section{Custo de produção}

O custo de produção de madeira cortada e processada pelo sistema semimecanizado foi de 5,16 $\mathrm{R} \$ \cdot \mathrm{m}^{-3}\left(2,34 \mathrm{US} \$ \cdot \mathrm{m}^{-3}\right)$ para o espaçamento $3 \times 2,5 \mathrm{~m}$, $4,66 \mathrm{R} \$ \cdot \mathrm{m}^{-3}\left(2, \mathrm{I}\right.$ I US\$· $\left.\mathrm{h}^{-1}\right)$ para o espaçamento $3 \times 3,33$

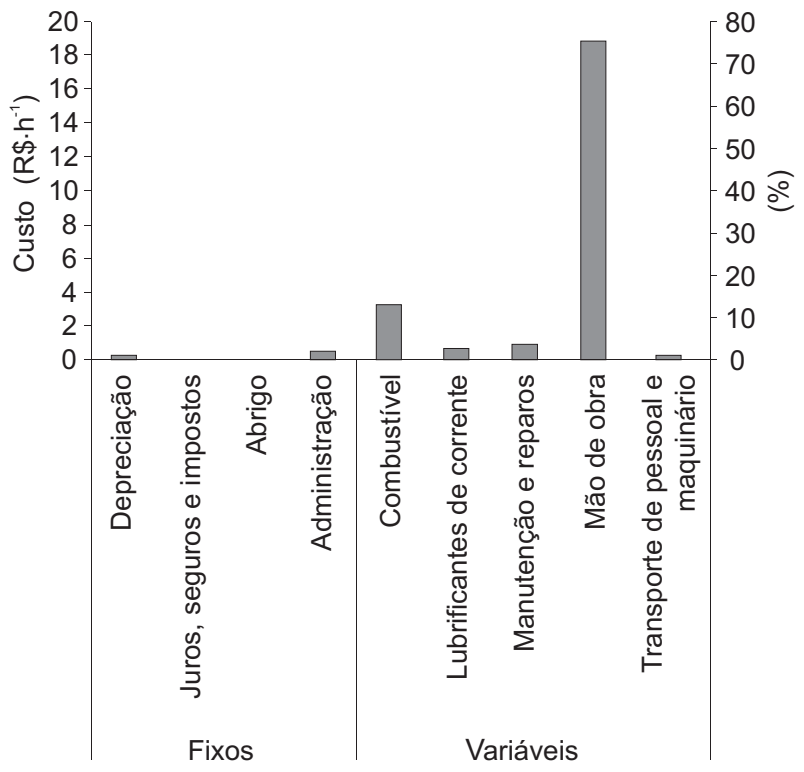

FIGURA 3 Análise de custo do sistema semimecanizado. FIGURE 3 Cost analysis of the semimechanized system.

e $4,22 \mathrm{R} \$ \cdot \mathrm{m}^{-3}\left(\mathrm{I}, 9 \mathrm{I}\right.$ US $\left.\$ \cdot \mathrm{m}^{-3}\right)$ para o espaçamento 3 x $4 \mathrm{~m}$. Observa-se a tendência de que o aumento do espaçamento reduz os custos de produção.

Os resultados são menores que os obtidos por Adreon (20I I), nos quais foi avaliada uma motosserra Stihl SM 36I, encontrando um custo de produção de 6,35 R\$.h $\mathrm{h}^{-1}$, em plantios de eucalipto no espaçamento $3 \times 3 \mathrm{~m}$. Esse fato está explicado pelos menores custos operacionais deste trabalho.

$\mathrm{Na}$ comparação com o sistema mecanizado, utilizando o harvester, Fernandes et al. (2013) e Leite et al. (20l3) encontraram em condições similares, maior custo de produção, fato esse que pode ser atribuído ao maior custo da máquina e por realizar outras operações como, descascamento e depósito da madeira em feixes.

\section{CONCLUSÕES}

Os maiores espaçamentos consumiram os menores tempos do ciclo operacional, especialmente para as atividades de deslocamento com máquina, traçamento e $1^{\circ}, 2^{\circ}$ e $3^{\circ}$ cortes.

Compreender a influência dos espaçamentos nas variações de produtividade do sistema semimecanizado pode auxiliar gestores florestais, considerando produtividade maior quando o espaçamento do plantio é aumentado.

O maior espaçamento resultou custos menores de produção e o custo da mão de obra foi o que teve maior influência sobre o custo operacional do sistema semimecanizado. 


\section{REFERÊNCIAS}

AMERICAN SOCIETY OF AGRICULTURAL ENGINEERS. ASAE D472-3: ASAE standards 200I: machinery, equipment and buildings: operating costs. Ames, 200I. 226 p.

ANDREON, B. C. Análise de custos do corte florestal semimecanizado em região declivosas no sul do Espírito Santo. 20Il. 24 f. Monografia (Graduação em Engenharia Florestal) - Universidade Federal do Espírito Santo, Jerônimo Monteiro, 20II.

CANTO, J. L. Colheita mecanizada de biomassa florestal para energia. 2009. 127 p. Tese (Doutorado em Ciência Florestal) - Universidade Federal de Viçosa, Viçosa, 2009.

FERNANDES, H. C.; BURLA, E. R.; LEITE, E. S.; MINETTE, L. J. Avaliação técnica e econômica de um "Harvester" em diferentes condições de terreno e produtividade da Floresta. Scientia Forestalis, Piracicaba, v. 4I, n. 97, p. $|4|-|5|, 20 \mid 3$.

LEITE, E. S.; FERNANDES, H. C.; MINETTE, L. J.; LEITE, H. G.; GUEDES, I. L. Modelagem técnica e de custos do Harvester no corte de madeira de eucalipto no sistema de toras curtas. Scientia Forestalis, Piracicaba, v. 4I, n. 98, p. 205-2I5, 2013.

MACHADO, C. C.; SILVA, E. N.; PEREIRA, R. S. O setor florestal brasileiro e a colheita florestal. In: MACHADO, C. C. (Org.). Colheita florestal. 2. ed. Viçosa, MG: UFV, 2008. p. 15-42.

MOREIRA, F.M. T. Análise técnica e econômica de subsistemas de colheita de madeira de eucalipto em terceira rotação. 2000. I48 p. Dissertação (Mestrado em Ciência Florestal) Universidade Federal de Viçosa, Viçosa, 2000.
MOUSAVI, R. Comparison of productivity, cost and environmental impacts of two harvesting methods in Northern Iran: short-log vs. long-log. 2009. 93 p. Dissertação (Master in Forest Sciences) - University of Joensuu, Joensuu, 2009.

PASSOS, C. A. M.; BUFULIN JUNIOR, L.; GONÇALVES, M. R. Avaliação silvicultural de Tectona grandis L.f., em Cáceres, MT, Brasil: resultados preliminares. Ciência Florestal, Santa Maria, v. I6, n. 2, p. 225-232, 2006.

SANT'ANNA, C. M. Corte florestal. In: MACHADO, C. C. (Ed.). Colheita florestal. 2. ed. Viçosa, MG: UFV, 2008. p. 66-9.

SANT'ANNA, C. M.; MALINOVSKI, J. R. Análise de fatores humanos e condições de trabalho de operadores de motosserra de Minas Gerais. Cerne, Lavras, v. 8, n. I, p. I|5-|2|, 2002

SANTOS, S. L. M.; MACHADO, C. C.; SOUZA, A. P.; LEITE, H. G. Avaliação técnica e econômica do corte florestal semimecanizado em diferentes volumes por árvore e comprimentos de tora. Revista Árvore, Viçosa, v. 24, n. 4, p. 4I7-422, 2000.

SILVA, E. N. Avaliação técnica e econômica do corte de pinus com harvester. 2008. 60 p. Dissertação (Mestrado em Ciência Florestal) - Universidade Federal de Viçosa, Viçosa, 2008.

SILVA, E. N.; MACHADO, C. C.; MINETTE, L. J.; SOUZA, A. P.; FERNANDES, H. C.; SILVA, M. L.; JACOVINE, L. A. Avaliação técnica e econômica do corte mecanizado de Pinus sp. com harvester. Revista Árvore, Viçosa, v. 34, n. 4, p. 745-753, 2010. 